\title{
Pulmonary Embolism Following Incomplete Surgical Resection of a Right Ventricular Myxoma: A Case Report and Review of the Literature
}

\author{
Yazan Assaf (ID) Maher Nasser $\cdot$ Hani Jneid $\cdot$ David Ott
}

Received: January 18, 2018 / Published online: April 17, 2018

(C) The Author(s) 2018

\begin{abstract}
Right ventricular (RV) myxomas are extremely rare, but may have dreadful clinical sequelae including pulmonary embolism (PE). We present a case of a patient who had an RV myxoma that was attached to the tricuspid valve, and therefore could not be resected completely during surgery, and remnants of the tumor were seen on transthoracic echocardiogram during post-operative follow-up.
\end{abstract}

Enhanced digital features To view enhanced digital features for this article go to https://doi.org/10.6084/ m9.figshare.6061451.

Y. Assaf $(\bowtie)$

Faculty of Medicine, Damascus University,

Damascus, Syria

e-mail: yazan.assaf.mail@gmail.com

M. Nasser · H. Jneid · D. Ott

Baylor College of Medicine, Houston, TX, USA

M. Nasser

Baylor St. Luke's Medical Center, Houston, TX, USA

M. Nasser · D. Ott

Texas Heart Institute, Houston, TX, USA

M. Nasser

Houston Methodist Hospital, Houston, TX, USA

H. Jneid

Michael E. DeBakey Veterans Affairs Medical Center, Houston, TX, USA

D. Ott

University of Texas Health Science Center at

Houston, Houston, TX, USA
Five months after surgery, the patient had PE, which could be due to tumor emboli or thromboemboli. Since repeat surgical resection was not feasible, the patient was started on warfarin. The patient is doing well and has had no PE recurrence over the past 20 months of follow-up. We have complemented the current case report with a comprehensive literature search and review on RV myxomas associated with PE in order to shed light on this uncommon but potentially lethal disorder. We concluded that right-sided cardiac myxomas, including RV myxomas, should be considered while dealing with PE, particularly in young patients with no risk factors, and that follow-up with echocardiography after surgery is important due to the possibility of recurrence, especially if complete resection was difficult to perform.

Plain Language Summary: Plain language summary available for this article.

Keywords: Myxoma; Pulmonary embolism; Right ventricle; Right ventricular myxoma

\section{PLAIN LANGUAGE SUMMARY}

Myxomas are a type of tumor that may occur inside heart chambers. Myxomas located in the right lower chamber of the heart (right ventricle) are very rare. However, such tumors may fragment and travel to the lung blood vessels. 
The patient in our case had a myxoma in the right ventricle. The tumor was attached to a valve in the heart. Partial removal of the tumor was performed so as not to damage the valve. Imaging after surgery showed remaining parts of the tumor. Five months later, the patient had clots in the lung blood vessels (pulmonary embolism). The patient was treated with a blood thinner. He is doing well since then. We searched the medical literature for cases of myxomas in the right ventricle of the heart associated with pulmonary embolism. We present a review of these cases along with our case. We concluded that myxomas of the right ventricle of the heart may be considered as a cause of pulmonary embolism. This is particularly true in young patients who do not have risk factors for pulmonary embolism. Our review also shows that imaging after surgery is important because myxomas may recur.

\section{INTRODUCTION}

Primary cardiac tumors are infrequent [1]. Myxoma is the most common type of such tumor in adults [2]. Most myxomas arise in the left atrium. Those located in the right ventricle are rare [3], and they are of a particular concern because of the risk of pulmonary tumor embolism. Right ventricular (RV) myxomas accompanied by pulmonary embolism (PE) are reported only in a limited number of studies in the medical literature [4-14]. Herein, we present a case of a young man who suffered from PE after incomplete surgical resection of an RV myxoma. To the best of our knowledge, this is the first report in the literature of confirmed PE following incomplete surgical resection of an RV myxoma. We also present a review of the literature pertaining to RV myxomas associated with PE in an effort to provide an insightful understanding of such cases.

\section{CASE PRESENTATION}

A 28-year-old male physician with no significant past medical history except for asthma, for which he takes albuterol and cetirizine, presented to a cardiology clinic with shortness of breath and palpitations for the last 3 months. The patient reported feeling 'the heart rubbing against the chest while leaning forward'. He denied chest discomfort or other cardiac symptoms. After auscultating his own chest, he heard a murmur for which he sought medical advice. Physical exam was normal except for a lowgrade holosystolic murmur best heard at the left lower sternal border, tachycardia and tachypnea with a respiratory rate of 25 . Electrocardiogram (ECG) showed sinus tachycardia with a heart rate of 112 beats per minute and right axis deviation. Transthoracic echocardiogram (TTE) revealed an RV mass (approximately $3 \times 2 \mathrm{~cm}$ ) attached to the interventricular septum by a small pedicle, dilated right heart chambers, moderate tricuspid regurgitation, and severe pulmonary hypertension (systolic pulmonary artery pressure $>60 \mathrm{mmHg}$ ) (Fig. 1). Cardiac magnetic resonance (MR) confirmed the presence of a well-circumscribed non-obstructive mass $(2.4 \times 1.9 \times 1.2 \mathrm{~cm})$ that appeared not to invade the surrounding cardiac structures located in the RV outflow tract, inferior to the pulmonary valve (Fig. 2). The patient denied a family history of cardiac tumors.

The patient underwent open heart surgery with median sternotomy and total cardiopulmonary bypass. Right ventriculotomy was performed, and the incision was extended superiorly and inferiorly. Traction sutures were placed on the right ventricle in order to improve exposure. A large and soft RV mass obstructing the RV outflow tract was identified in the right ventricle attached to the anterosuperior and septal leaflets of the tricuspid valve and partially attached to the interventricular septum. Based on the surgeon's decision, the mass was partially excised without compromising the integrity of the tricuspid valve (Fig. 3). TEE was used to evaluate the valve function and revealed mild tricuspid regurgitation. The patient had uneventful surgical recovery and was discharged following a 5-day hospitalization period.

The pathology report of the resected mass (two specimens) confirmed the diagnosis of myxoma. The first specimen consisted of three 

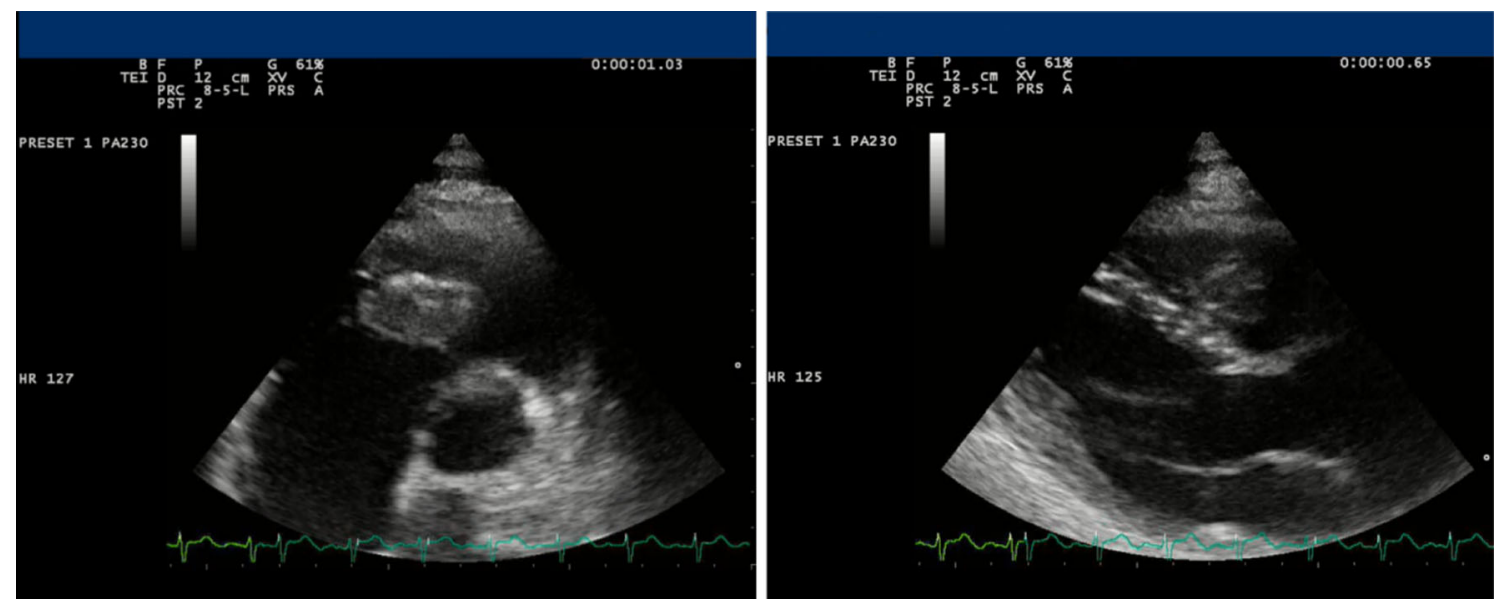

Fig. 1 Transthoracic echocardiogram (TTE) views before surgery showing a right ventricular mass (approximately $3 \times 2 \mathrm{~cm}$ ) attached to the interventricular septum by a small pedicle and dilated right heart chambers

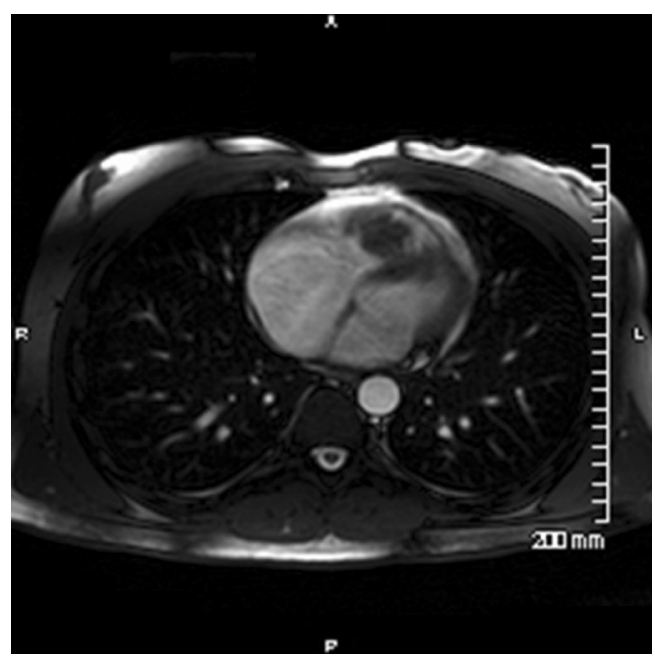

Fig. 2 Cardiac magnetic resonance (MR) before surgery revealing a well-circumscribed non-obstructive mass $(2.4 \times 1.9 \times 1.2 \mathrm{~cm})$ that appeared not to invade the surrounding cardiac structures located in the right ventricular outflow tract, inferior to the pulmonary valve

fragments of tissue measuring $1.3 \times 1 \times 0.5 \mathrm{~cm}$ in aggregate. One portion of tissue had a tanwhite base with tan-red tissue attached and projecting from the base. Another portion of tissue was predominately tan-red with focal yellow tissue, reminiscent of adipose tissue. The third portion of tissue was tan-red. The second specimen was an aggregate of pink-tan to gray-white, rubbery, ragged, and soft tissue fragments measuring $3.5 \times 3.5 \times 1.5 \mathrm{~cm}$.

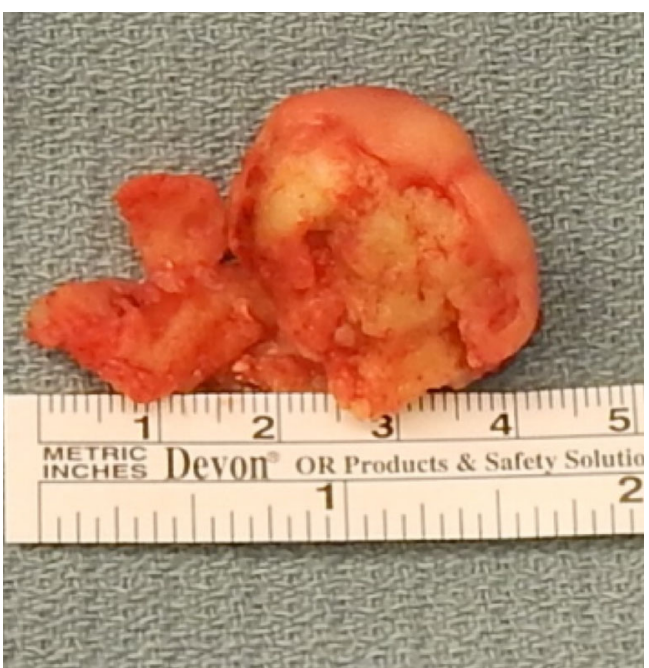

Fig. 3 The resected myxoma (predominantly tan-red with focal yellow tissue measuring about $4 \times 3 \times$ $1.5 \mathrm{~cm}$ )

Sectioning revealed a gray-white to yellow-green, rubbery and friable cut surface. No firm areas were identified. Microscopically, the majority of the first specimen consisted of fibrin clot. There was a fragment of bland polygonal to spindle-shaped cells within a myxoid matrix. Factor VIII showed focal positivity within the bland cells and was positive in capillaries. CD34 was positive in capillaries. No entrapped cores of elastic tissue were seen on Verhoeff-Van Gieson stain (elastin stain). Trichrome stain 


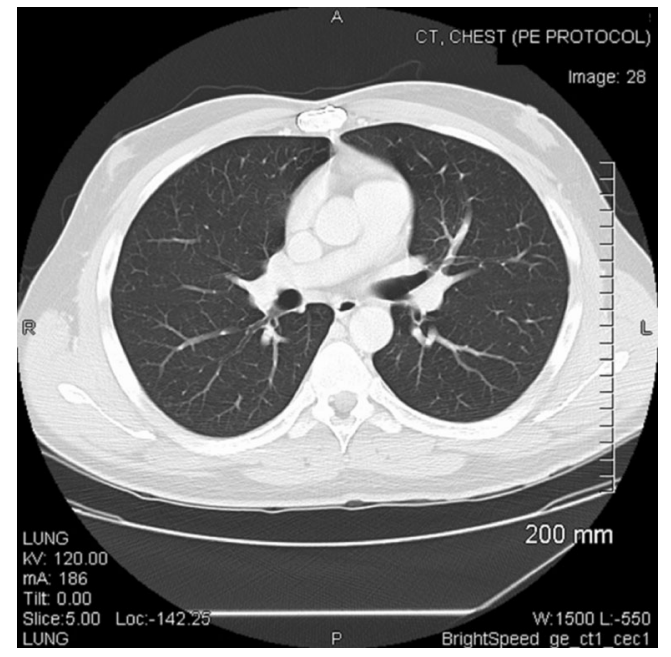

Fig. 4 Chest computerized tomography (CT) after surgery scan showing multiple emboli in the right lower lobe segmental and subsegmental pulmonary arterial branches, as well as peripheral airspace disease in the right lower lobe suggestive of pulmonary infarction

showed no laminated elastic fibers within the matrix. The morphologic and immunohistochemical findings were compatible with cardiac myxoma. The microscopic examination of the second specimen showed myxoma cells with an oval nucleus which were forming ring structures around small thin-walled blood vessels. An accompanying mononuclear inflammatory cell infiltrate was present. Hemosiderin-laden macrophages were also present. A myxoid background was focally present. The majority of the tissue showed secondary changes of fibrosis and calcification which obscured the underlying nature of the lesion. The myxoma was attached to a portion of benign cardiac muscle.

Two weeks after surgery, a follow-up TTE showed a residual RV mass (approximately $2 \times 1.5 \mathrm{~cm}$ ) attached to the interventricular septum.

Five months after surgery, the patient presented to the emergency department with rightsided pleuritic chest pain and upper abdominal discomfort. The patient took 15 airplane flights over the previous 2 months, some of which were very long. He was tachypneic (22 breaths/ min), and his ECG showed sinus tachycardia with a heart rate of $118 \mathrm{bpm}$ and right bundle branch block (RBBB). Serum D-dimer was elevated $(0.9 \mathrm{mg} / \mathrm{l})$. His chest X-ray was normal. A chest computerized tomography (CT) scan revealed multiple emboli in the right lower lobe segmental and subsegmental pulmonary arterial branches, as well as peripheral airspace disease in the right lower lobe suggestive of pulmonary infarction (Fig. 4). Venous Doppler ultrasound of the legs showed bilateral patent and compressible venous systems and no evidence of thrombus. TTE and transesophageal echocardiogram (TEE) were done and reported dilation of the inferior vena cava (IVC), right atrium and right ventricle and a mass (approximately $2 \times 1.5 \mathrm{~cm}$ ) attached to the base of the RV wall just below the insertion of the anterior tricuspid leaflet (Fig. 5). The tricuspid jet was insufficient to calculate pulmonary pressure. Cardiac MR showed a small residual tissue $(9 \times 9 \mathrm{~mm})$ adjacent to the interventricular septum and another residual tissue $(7 \times 9 \mathrm{~mm})$ attached to the RV free wall (Fig. 6). The patient was diagnosed with PE, due to either tumor emboli or thromboemboli, and was started on warfarin. On further follow-up (20 months following PE), he had no recurrent PE.

Informed consent was obtained from the patient for being included in the study.

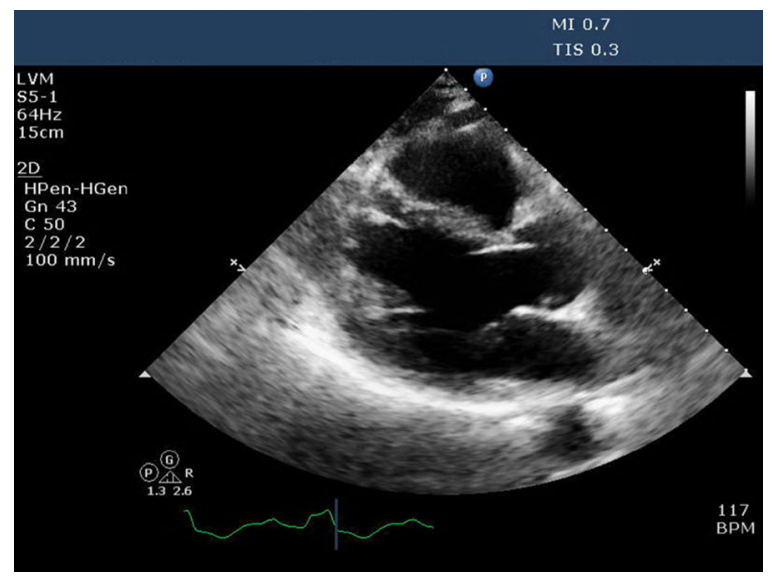

Fig. 5 Transesophageal echocardiogram (TEE) after surgery revealing dilation of the inferior vena cava (IVC), right atrium and right ventricle and a mass (approximately $2 \times 1.5 \mathrm{~cm}$ ) attached to the base of the right ventricular wall just below the insertion of the anterior tricuspid leaflet 

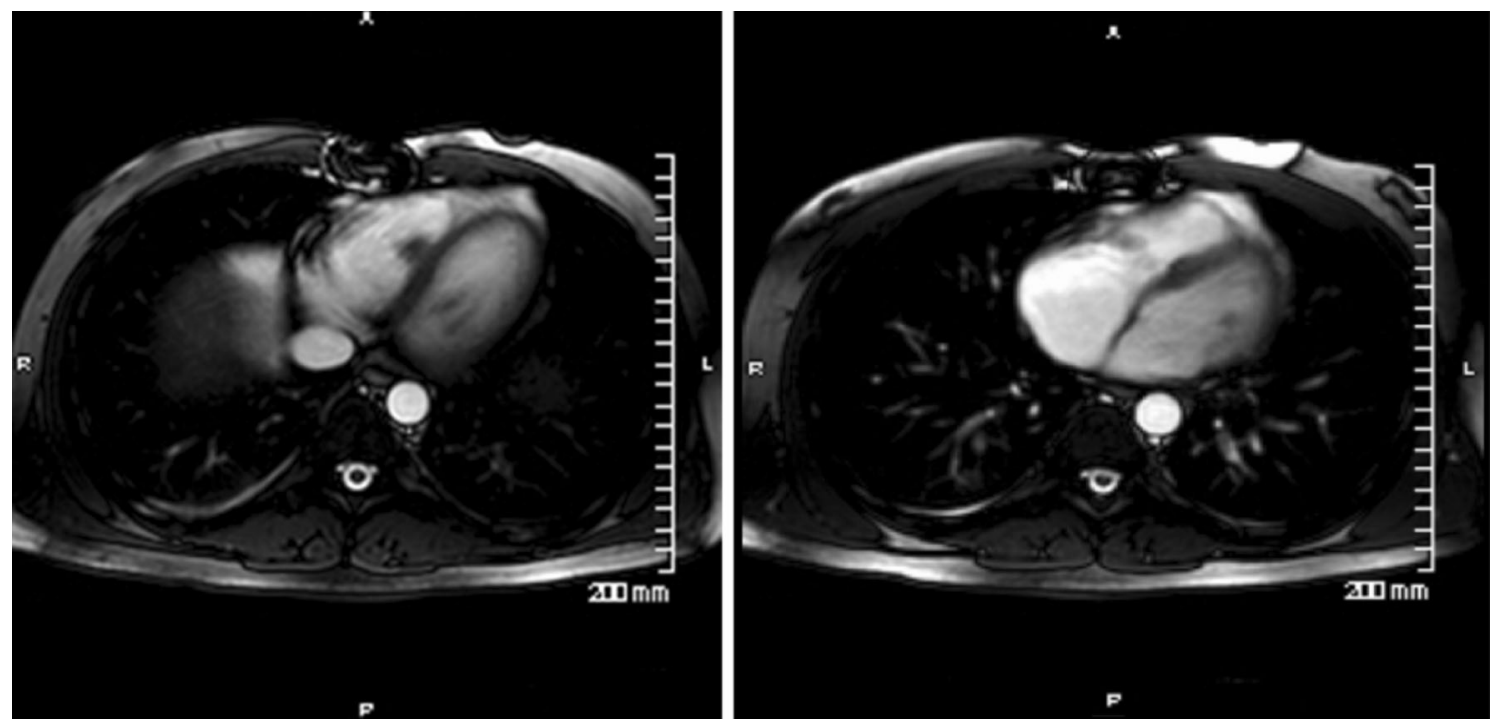

Fig. 6 Cardiac magnetic resonance (MR) views after surgery showing a small residual tissue $(9 \times 9 \mathrm{~mm})$ adjacent to the interventricular septum and another residual tissue $(7 \times 9 \mathrm{~mm})$ attached to the right ventricular free wall

\section{DISCUSSION}

In the 18th century, the French physician, DeSénac, stated, "The heart is an organ too noble to be attacked by a primary tumor" $[15,16]$. We now know that primary tumors of the heart do exist, although they are rare with a frequency of just about $0.02 \%$ [1]. Secondary tumors of the heart are approximately 30 -fold more common than primary ones [17]. Around $75 \%$ of primary cardiac tumors are benign. Myxomas, which constitute about half of the benign cardiac tumors, are the most common primary tumors of the heart in adults, while rhabdomyomas predominate in children $[2,18]$. Myxomas are more common in women and are usually diagnosed between the third and sixth decades of life [19]. Both TTE and TEE play important roles in the initial diagnosis of cardiac myxomas, with CT and cardiac MR being supplemental [18]. It was remarkable that preoperative TTE in our case revealed severe pulmonary hypertension. We believe that could be due to recurrent pulmonary emboli that went unnoticeable. Postoperative TTE in our case revealed dilation of the IVC, right atrium and right ventricle, which we believe could be due to the mild TR revealed on the TEE done during the surgery after excising the mass. We think that the mass was distorting the tricuspid valve.

Most cardiac myxomas are sporadic. Only about $7 \%$ of them are hereditary and occur along with skin pigmentation and endocrinopathy in an autosomal dominant disorder called Carney Complex [20]. Our patient denied any family history of cardiac tumors.

Three-quarters of cardiac myxomas are located in the left atrium. Most of the rest occur in the right atrium. RV myxomas were reported in just $3-4 \%$ of cardiac myxoma cases [3]. Myxomas range between 1 and $15 \mathrm{~cm}$ in diameter weighing between 15 and $180 \mathrm{~g}$ [21]. Cardiac myxomas are usually pedunculated and have a smooth, villous or friable surface. Smooth myxomas are known for obstructive complications, whereas villous and friable ones tend to cause embolic complications [22]. The myxoma in the case we are presenting was friable according to the pathology report. This, in addition to the absence of deep vein thrombosis, favors the diagnosis of tumor emboli in our patient.

Location, size, and mobility of cardiac myxomas determine their clinical presentation. Embolism, obstruction, and constitutional symptoms are common manifestations. 
Embolism occurs in $30-40 \%$ of cases. Systemic embolism is much more common than PE, as most myxomas are predominantly located in the left atrium. Only few cases of RV myxomas associated with PE were reported [4-14].

Once a cardiac myxoma is diagnosed, it should be surgically resected to avoid its associated complications. Recurrence rate is less than $3 \%$ in sporadic cases, but ranges between 12 and 22\% in familial ones. Factors associated with recurrence include multicentricity, young age, familial cases, and incomplete surgical resection. Follow-up is usually done after surgery using echocardiography [18]. In our case, the tumor could not be completely resected due to its attachment to the tricuspid valve.

Pulmonary tumor emboli and thromboemboli are not easily differentiable. CT scans and pulmonary angiograms are often nonspecific for tumor emboli. Tumor emboli usually display a more peripheral distribution in the subsegmental arteries (as likely occurred in our patient) [23]. On the other hand, thromboemboli are associated with long and multiple airplane flights [24]. Therefore, the fact that the patient in the case we are reporting had taken numerous and long flights before he suffered from PE raises the possibility that thromboembolism contributed to the PE. Thromboemboli are also correlated with elevation of serum D-dimer [25], which was high when the PE was diagnosed in the case we are reporting. Nevertheless, the elevation in D-dimer was slight and lower extremities venous Doppler showed no evidence of thrombi. Pulmonary thromboemboli are more frequently correlated with higher serum D-dimer levels [26].

Confirmation of pulmonary tumor emboli require a lung biopsy procedure, which can be done with either open-lung or transbronchial approaches [27]. A definitive diagnosis of pulmonary tumor emboli through invasive procedures was not pursued in our patient since surgery was already done, and the patient was started on warfarin. The fact that no recurrence of PE happened during the follow-up may favor but does not confirm that the emboli were thrombotic in origin.

Echo-guided percutaneous trans-catheter biopsy of intracardiac masses, especially in the right-sided chambers, is safe and can accurately provide histopathological diagnosis. Such an approach may therefore mitigate the need for surgical excisional biopsy and potentially alter clinical management in a large number of patients [28].

We conducted a search of published cases describing RV myxomas associated with PE in PubMed using the following search terms: pulmonary embolism, myxoma, ventricular, right ventricular myxoma. The search yielded 29 articles, 11 of which were pertaining to RV myxomas associated with PE. We present a review of these 11 cases along with the case we are presenting (Table 1 ).

The oldest case was published in 1971 [14], and the most recent one, apart from ours, in 2016 [4]. Singh el al. reported two cases of cardiac masses in his article, however only one of them was confirmed as a myxoma and included in our review [10]. Eight of the 11 cases were reported in English language [4-8, 10, 12, 13]. Urina Triana et al. reported their case in Spanish language. We included it since we could translate the entire article [11]. The case reported by Vernant et al. was in French language. We translated and included the abstract of that article as it was the only part we found [14]. Tatebayashi et al. reported their case in Japanese language. We only included the abstract of that article since it was available in English language [9].

The number of male and female patients was equal. The mean age of patients was approximately 33 years, and while the youngest patient was 12 years old [13], the oldest one was 76 years old [9]. However, most patients were in their 20 or 30s.

Although the presentation was not the same for all the cases, eight cases showed that pulmonary embolism could be the first presentation of RV myxomas [4-9, 11, 12]. This indicates the significance of performing echocardiography in cases of unexplained PE especially in young patients who do not have risk factors of PE.

A summary of the most frequent findings of the cases in our review, including our case, is shown in Table 2. 


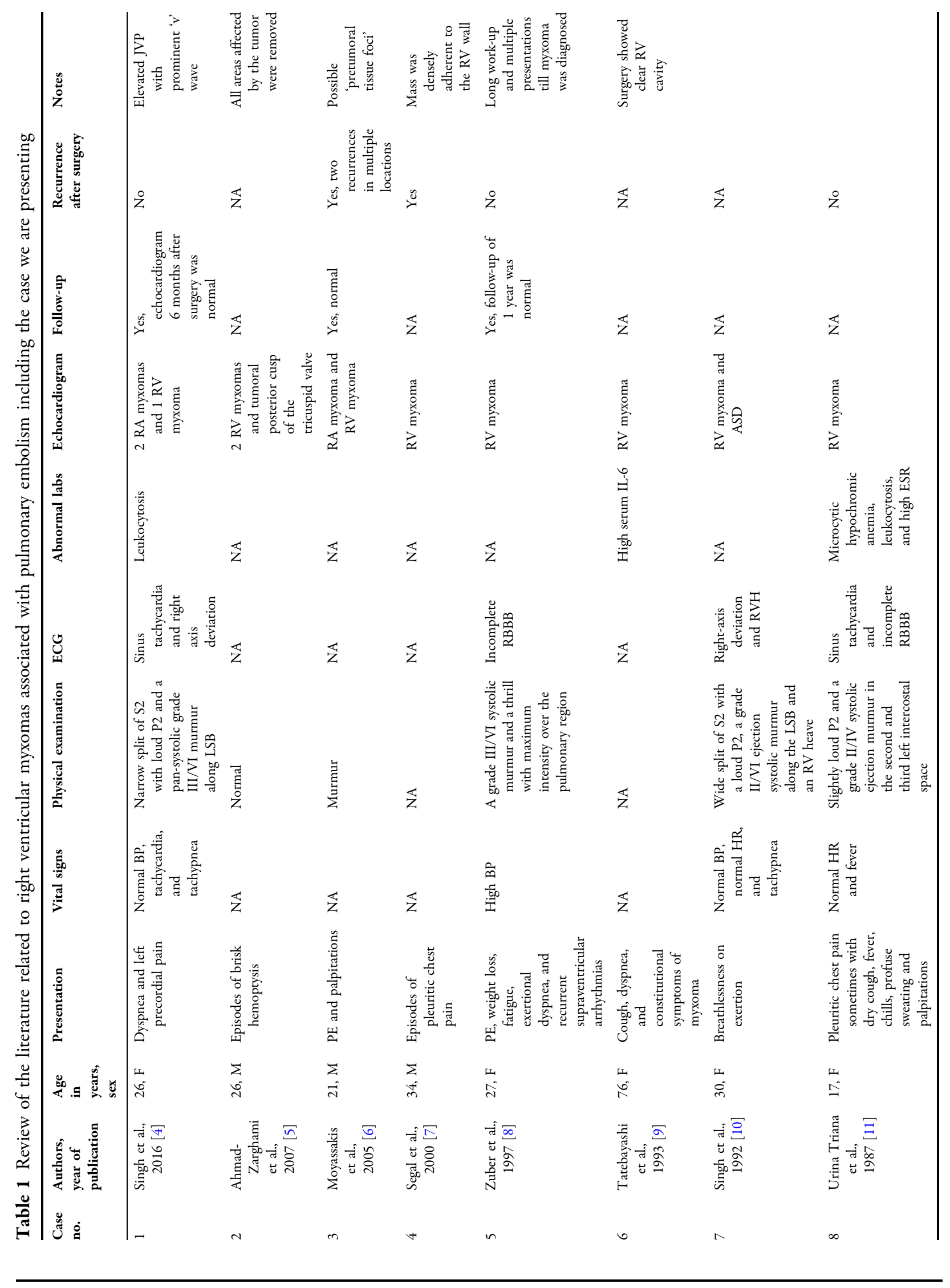




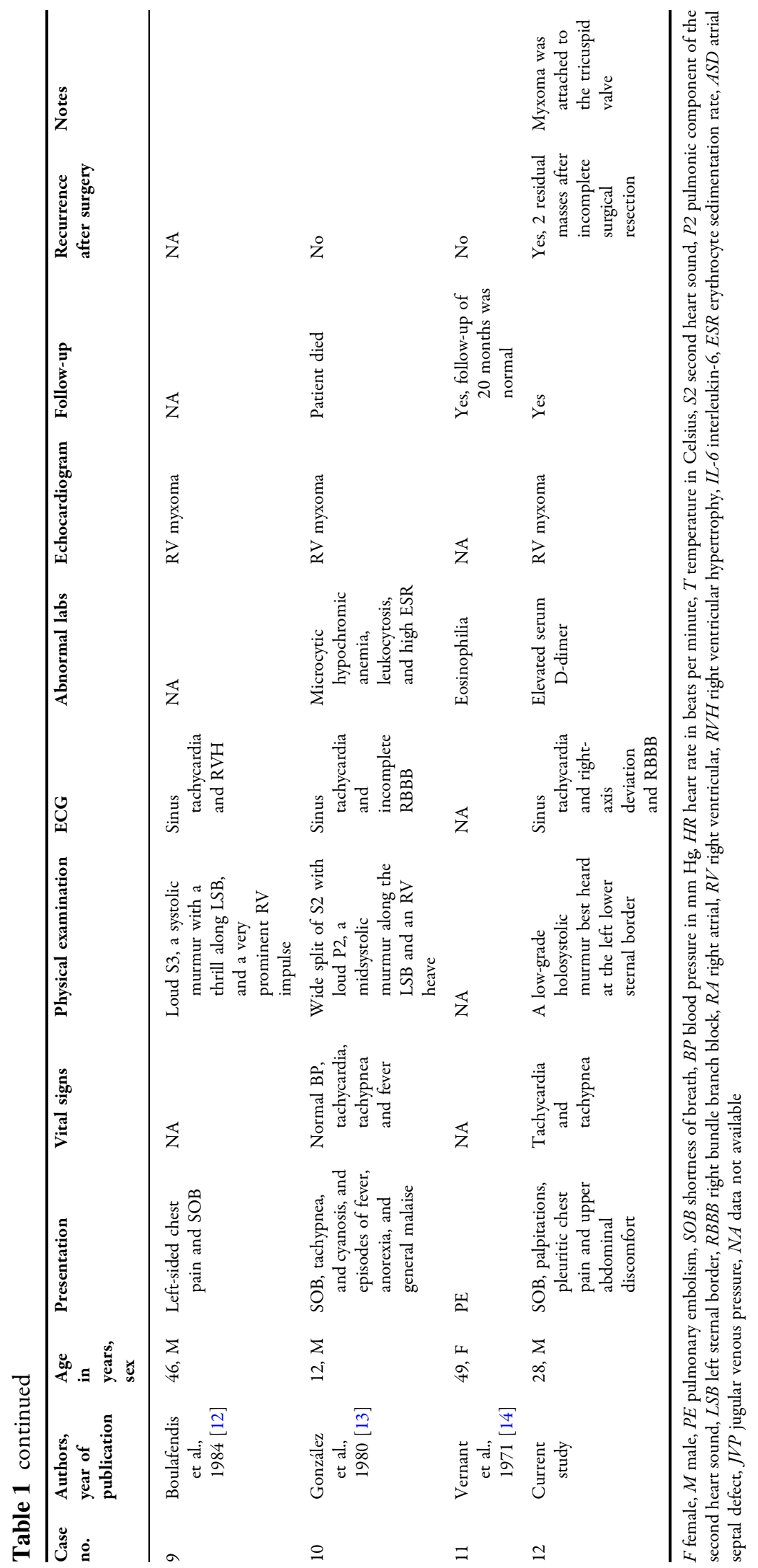


Table 2 Most frequent findings of the cases in the review of literature including our case

\begin{tabular}{|c|c|}
\hline Finding & $\begin{array}{l}\text { No. of cases that reported } \\
\text { the finding }\end{array}$ \\
\hline \multicolumn{2}{|l|}{ Signs and symptoms } \\
\hline SOB & $7[4,8-10,12,13]^{\mathrm{a}}$ \\
\hline $\mathrm{CP}$ & $5[4,7,11,12]^{a}$ \\
\hline Palpitations & $3[6,11]^{\mathrm{a}}$ \\
\hline \multicolumn{2}{|l|}{ Vital signs } \\
\hline Tachypnea & $4[4,10,13]^{a}$ \\
\hline Tachycardia & $3[4,13]^{a}$ \\
\hline Fever & $2[11,13]$ \\
\hline \multicolumn{2}{|l|}{ Physical examination } \\
\hline Murmur & $8[4,6,8,10-13]^{a}$ \\
\hline Loud P2 & $4[4,10,11,13]$ \\
\hline RV heave & $3[10,12,13]$ \\
\hline Wide split of S2 & $2[10,13]$ \\
\hline \multicolumn{2}{|l|}{ ECG } \\
\hline Sinus tachycardia & $5[4,11-13]^{a}$ \\
\hline RBBB & $4[8,11,13]^{a}$ \\
\hline Right axis deviation & $3[4,11]^{a}$ \\
\hline RVH & $2[10,12]$ \\
\hline \multicolumn{2}{|l|}{ Labs } \\
\hline Leukocytosis & $3[4,11,13]$ \\
\hline $\begin{array}{l}\text { Microcytic } \\
\text { hypochromic anemia }\end{array}$ & $2[11,13]$ \\
\hline High ESR & $2[11,13]$ \\
\hline
\end{tabular}

$S O B$ shortness of breath, $S 2$ second heart sound, $P 2$ pulmonic component of the second heart sound, $R B B B$ right bundle branch block, $R V H$ right ventricular hypertrophy, ESR erythrocyte sedimentation rate

${ }^{a}$ Including our case

Echocardiography was remarkable in four cases $[4-6,10]$. It revealed two right atrial masses and one RV mass in the first one [4], two RV masses and tumoral posterior cusp of the tricuspid valve in the second one [5], one right atrial mass and one RV mass in the third one [6], and one RV myxoma and atrial septal defect in the fourth one [10].

Recurrence of myxoma after surgical resection happened in the case reported by Moyassakis et al. Therefore, the patient underwent a second surgical resection, after which recurrence happened again [6]. Segal et al. reported a case in which the mass was densely adherent to the wall of the right ventricle. Surgical resection was performed, however recurrence occurred [7]. These two cases in addition to the case we are presenting show that recurrence of myxomas may happen. Recurrence seems to occur particularly when complete resection of the mass is not possible. This emphasizes the importance of the follow-up including echocardiography after surgery. It was notable that only four of the 11 cases we reviewed reported follow-up $[4,6,8,14]$.

Zuber et al. reported a patient who presented with PE several times after a ligament reconstruction surgery. At first, the patient was only treated with anticoagulation. Echocardiography, which showed RV myxoma, was not performed until the patient presented for the third time [8]. The case reported by Boulafendis et al. also shows that echocardiogram was performed during a second presentation after only giving anticoagulation therapy during the first one [12]. González et al. reported the death of a 12-year-old boy after failing to diagnose the myxoma early [13]. These cases show that the diagnosis of myxomas can be easily missed in case of not suspecting them.

Singh et al. reported an RV myxoma seen on echocardiogram. Nonetheless, the RV cavity was clear during surgery. Severe right heart failure occurred in the immediate postoperative period, and embolism of the tumor was suspected [10].

\section{CONCLUSIONS}

Despite being rare, cardiac tumors including RV myxomas should be included in the differential of the occasional patient with unexplained PE. This is particularly important when there are no risk factors or predisposing etiologies for PE and 
the patient is young. The presentation of RV myxomas does not seem to be specific, and the diagnosis is usually accidentally made on a TTE obtained to examine RV strain or failure. Furthermore, following up patients with echocardiography after surgery is important since recurrence, which seems to be related to incomplete resection, could happen.

\section{ACKNOWLEDGEMENTS}

We thank the participant of this study.

Funding. No funding or sponsorship was received for this study or publication of this article.

Authorship. All named authors meet the International Committee of Medical Journal Editors (ICMJE) criteria for authorship for this article, take responsibility for the integrity of the work as a whole, and have given their approval for this version to be published.

Disclosures. Yazan Assaf, Maher Nasser, Hani Jneid, and David Ott have nothing to disclose.

Compliance with Ethics Guidelines. Informed consent was obtained from the patient for being included in the study.

Data Availability. Data sharing is not applicable to this article as no datasets were generated or analyzed during the current study.

Open Access. This article is distributed under the terms of the Creative Commons Attribution-NonCommercial 4.0 International License (http://creativecommons.org/licenses/ by-nc/4.0/), which permits any noncommercial use, distribution, and reproduction in any medium, provided you give appropriate credit to the original author(s) and the source, provide a link to the Creative Commons license, and indicate if changes were made.

\section{REFERENCES}

1. Reynen K. Frequency of primary tumors of the heart. Am J Cardiol. 1996;77(1):107.

2. Jain S, Maleszewski JJ, Stephenson CR, Klarich KW. Current diagnosis and management of cardiac myxomas. Expert Rev Cardiovasc Ther. 2015;13(4):369-75.

3. Gribaa R, Slim M, Kortas C, et al. Right ventricular myxoma obstructing the right ventricular outflow tract: a case report. J Med Case Rep. 2014;8:435.

4. Singh S, Tripathy MP, Mohanty BB, Biswas S. Sporadic multicentric right atrial and right ventricular myxoma presenting as acute pulmonary thromboembolism. Heart Views. 2016;17(1):19-22.

5. Ahmadi-Zarghami H, Fallah-Tafti S, Behzadnia N, Sharif-Kashani B, Karimi S, Eslampanah S. Photoclinic. Right ventricular myxoma presenting as pulmonary embolism. Arch Iran Med. 2007;10(2):268, 70-1.

6. Moyssakis I, Anastasiadis G, Papadopoulos D, Margos P, Votteas V. Second recurrence of cardiac myxoma in a young patient. A case report. Int J Cardiol. 2005;101(3):501-2.

7. Segal OR, Robinson NM, Timmis AD. Images in cardiology: recurrent myxoma of the right ventricle. Heart. 2000;84(6):652.

8. Zuber M, Evequoz D, Stulz P, Erne P. Right ventricular myxoma mimicking recurrent pulmonary embolism after primary ligament reconstruction. Vasa. 1997;26(1):49-51.

9. Tatebayashi T, Onuki T, Ito H, Kaguraoka H, Sakasegawa M, Nitta S. Right ventricular myxoma with near obstruction of the pulmonary artery. Nihon Kyobu Geka Gakkai Zasshi. 1993;41(11):2256-60.

10. Singh B, Kaul UA, Jolly N, Khalilullah M. Pulmonary embolisation of right-sided intracardiac masses-report of two cases. Indian Heart J. 1992;44(4):241-3.

11. Urina Triana MA, Guadarrama JL, Febles TM, et al. Myxoma of the right ventricle: report of a clinical case and review of the literature. Arch Inst Cardiol Mex. 1987;57(1):51-5.

12. Boulafendis D, Heine J, Samaan HA. Right ventricular myxoma. Int J Cardiol. 1984;5(2):216-9.

13. Gonzalez A, Altieri PI, Marquez EU, Cox RA, Castillo M. Massive pulmonary embolism associated with a right ventricular myxoma. Am J Med. 1980;69(5):795-8. 
14. Vernant P, Aigueperse J, Gaudeau S, Lellouche D, Oustrieres G. Myxoma of the right ventricle revealed by repeated pulmonary embolisms. Arch Mal Coeur Vaiss. 1971;64(4):609.

15. Yusuf N. Is the heart truly noble? South Asian J Cancer. 2013;2(2):80-2.

16. Bowman IA. Jean-Baptiste Senac and his treatise on the heart. Tex Heart Inst J. 1987;14(1):5-11.

17. Paraskevaidis IA, Michalakeas CA, Papadopoulos $\mathrm{CH}$, Anastasiou-Nana M. Cardiac tumors. ISRN Oncol. 2011;2011:208929.

18. Reynen K. Cardiac myxomas. $\mathrm{N}$ Engl J Med. 1995;333(24):1610-7.

19. Wang Z, Chen S, Zhu M, et al. Risk prediction for emboli and recurrence of primary cardiac myxomas after resection. J Cardiothorac Surg. 2016;11:22.

20. Amano J, Kono T, Wada Y, et al. Cardiac myxoma: its origin and tumor characteristics. Ann Thorac Cardiovasc Surg. 2003;9(4):215-21.

21. Pinede L, Duhaut P, Loire R. Clinical presentation of left atrial cardiac myxoma. A series of 112 consecutive cases. Medicine (Baltimore). 2001;80(3):159-72.
22. Thyagarajan B, Kumar MP, Patel S, Agrawal A. Extracardiac manifestations of atrial myxomas. J Saudi Heart Assoc. 2017;29(1):37-43.

23. Roberts KE, Hamele-Bena D, Saqi A, Stein CA, Cole RP. Pulmonary tumor embolism: a review of the literature. Am J Med. 2003;115(3):228-32.

24. Sajid MS, Desai M, Morris R, Hamilton G. Kneelength graduated compression stockings for thromboprophylaxis in air travellers: a meta-analysis. Int J Angiol. 2008;17(3):119-23.

25. Brill-Edwards P, Lee A. D-dimer testing in the diagnosis of acute venous thromboembolism. Thromb Haemost. 1999;82(2):688-94.

26. Hochuli M, Duewell S, Frauchiger B. Quantitative D-dimer levels and the extent of venous thromboembolism in CT angiography and lower limb ultrasonography. Vasa. 2007;36(4):267-74.

27. Schriner RW, Ryu JH, Edwards WD. Microscopic pulmonary tumor embolism causing subacute cor pulmonale: a difficult antemortem diagnosis. Mayo Clin Proc. 1991;66(2):143-8.

28. Reddy G, Maor E, Bois MC, et al. Percutaneous transcatheter biopsy for intracardiac mass diagnosis. EuroIntervention. 2017;13(12):e1436-43. 